\title{
Chemical reaction in MHD flow past a vertical plate with mass diffusion and constant wall temperature with hall current
}

\author{
U. S. Rajput and Neetu Kanaujia* \\ Department of Mathematics and Astronomy, University of Lucknow, INDIA \\ "Corresponding Author: e-mail: rajputneetulko@gmail.com
}

\begin{abstract}
Chemical reaction plays an important role in MHD flow. It has industrial applications, such as design of chemical processing equipments, food processing and cooling towers etc. In the present paper, chemical reaction effect on a viscous, incompressible and electrically conducting fluid with unsteady MHD flow past an impulsively started oscillating vertical plate with variable mass diffusion and constant wall temperature is studied. The solution of the model is obtained by Laplace transform method. The effect of parameters is shown with the help of graphs. The skin-friction and Sherwood number have been tabulated. It has been observed that chemical reaction has decreasing effect on fluid velocity. Our study gets motivation from the study of Narayanan et al. (2016), which has similar findings on chemical reaction with a slightly different fluid model.
\end{abstract}

Keywords: Chemical reaction, MHD, Hall current, Skin friction, and Sherwood number.

DOI: http://dx.doi.org/10.4314/ijest.v8i4.4

\section{Introduction}

The problems related to chemical reaction, Hall current, heat transfer and mass diffusion are of great interest due to their applications in many processes. From the industrial point of view, oscillatory flow is always important, for it has many practical applications. Energy (heat and mass) transfer is used in various engineering and science problems. Chemical reactions are used in the field of Psychrometer to measure humidity, paper production, glass blowing, metal spinning, food processing, and polymer solution, and also in various fluid flows related to engineering problems. These processes abound in metallurgical engineering as well as chemical engineering. They could also be found in solar collectors, nuclear reactor safety as well as combustion systems. The Magneto hydro dynamic power generation is one of the examples of new and unique method of electricity generation. The MHD generators are useful to power submarines aircraft and electrical power production for domestic applications. They can also be used as power plants in industry and uninterrupted power supply system and power generation in space craft. Defense applications, experiments in hypersonic wind tunnels, MHD-oriented power generation are in superior phase these days and near commercialization.

Some related research works are mentioned here. Beg and Ghosh (2010) investigated magneto hydrodynamic flow with oscillatory surface temperature. Datta et al. (1976) have studied oscillatory MHD over a plate. Sato (1961) analyzed the Hall effect on ionized gas between parallel plates. Kumar et al. (2016) have studied effect of viscous dissipation on mixed convection flow in a vertical double passage channel using Robin boundary conditions. Yamanishi (1962) has studied MHD flow between two parallel plates. Ibrahim (2014) and Ibrahim et al. (2010) have studied effects of chemical reaction on two different models. Chambre et al. (1958) have studied the diffusion of a chemically reactive species in a laminar boundary layer flow. Dash et al. (2012) have analyzed heat transfer in viscous free convective fluctuating MHD flow through porous media past a vertical porous plate with variable temperature. Rajput and Kumar (2016) have studied magneto hydrodynamic flow over exponentially accelerated plate. Earlier, we (2016) have studied MHD flow past over a vertical plate with mass diffusion. In the present model, 
we are considering chemical reaction effect on MHD flow over an impulsively started oscillating vertical plate with variable mass diffusion and constant wall temperature with Hall current.

\section{Mathematical analysis}

The physical model is given in Figure 1.

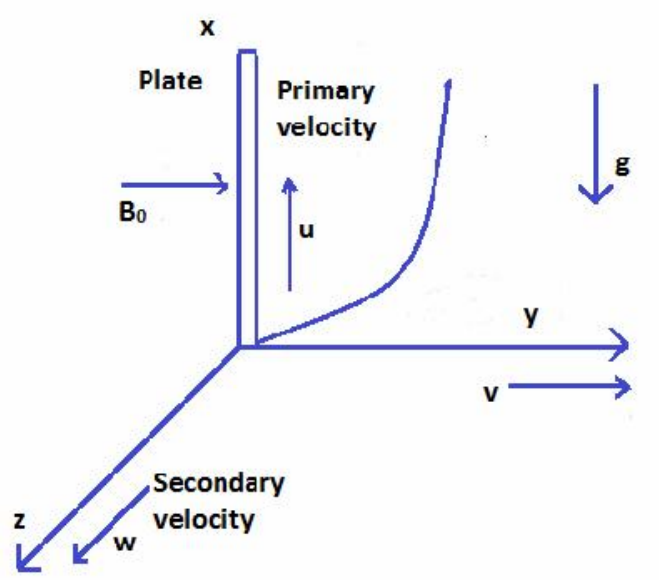

Figure 1 - Physical model

The flow is unsteady and incompressible. The $\mathrm{x}$ axis is considered along the fluid motion. Magnetic field $B_{0}$ of uniform strength is taken perpendicular to x axis. Initially it has been considered that the fluid and the plate are at the same temperature $\left(T_{\infty}\right)$. $C_{\infty}$ is the species concentration within the fluid. The plate starts oscillating after $t=0$ with frequency $\omega$. The species concentration and temperature of the plate are raised to $C_{w}$ and $T_{w}$, respectively. The force due to inertia is neglected. It is also relevant to mention the studies of Nguyen et al. (2016) and Takabi et al. (2014). The fluid model with Boussinesq approximations is as under:

$$
\begin{aligned}
& \frac{\partial u}{\partial t}=v \frac{\partial^{2} u}{\partial y^{2}}+g \beta\left(T-T_{\infty}\right)+g \beta^{*}\left(C-C_{\infty}\right)-\frac{\sigma B_{0}{ }^{2}}{\rho\left(1+m^{2}\right)}(u+m w), \\
& \frac{\partial w}{\partial t}=v \frac{\partial^{2} w}{\partial y^{2}}-\frac{\sigma B_{0}{ }^{2}}{\rho\left(1+m^{2}\right)}(w-m u), \\
& \frac{\partial C}{\partial t}=D \frac{\partial^{2} C}{\partial y^{2}}-K_{C}\left(C-C_{\infty}\right), \\
& \rho C_{p} \frac{\partial T}{\partial t}=k \frac{\partial^{2} T}{\partial y^{2}} .
\end{aligned}
$$

The boundary conditions are as under.

$$
\begin{aligned}
& t \leq 0: u=0, w=0, T=T_{\infty}, C=C_{\infty}, \text { for every } \mathrm{y} \\
& t>0: u=u_{0} \cos \omega t, w=0, T=T_{w}, C=C_{\infty}+\left(C_{w}-C_{\infty}\right) \frac{u_{0}{ }^{2} t}{v} \text { at } \mathrm{y}=0 \\
& u \rightarrow 0, w \rightarrow 0, C \rightarrow C_{\infty}, \mathrm{T} \rightarrow T_{\infty} \text { as } \mathrm{y} \rightarrow \infty
\end{aligned}
$$

The symbols used are as under $u$ - fluid velocity along $x$ - direction, $w$ - fluid velocity along $z$ - direction, $m$ - the Hall parameter, $g$ - gravity, $\beta$ - vol. coeff. of thermal expansion, $\beta^{*}$ - vol. coeff. of concentration expansion, $t$ - time, $C$ - species concentration in the fluid , $C_{w}-$ species 
concentration at the plate, D - diffusion coeff., $T_{w}$ - temperature of the plate, $T$ - the temperature of the fluid , $\mathrm{k}$ - the thermal conductivity, $v$ - the kinematic viscosity, $\rho$ - the fluid density, $K_{c}$-chemical reaction parameter, $\sigma$ - electrical conductivity, the magnetic permeability, and $C_{P}$ is specific heat at constant pressure. Here $m=\omega_{e} \tau_{e}$ with $\omega_{e}$ - cyclotron frequency of electrons and $\tau_{e}$ - electron collision time.

To write the equations (1) - (4) in non- dimensional from, we introduce the following quantities:

$$
\begin{aligned}
& \bar{u}=\frac{u}{u_{0}}, \bar{w}=\frac{w}{u_{0}}, \bar{y}=\frac{y u_{0}}{v}, S c=\frac{v}{D}, P_{r}=\frac{\mu C_{P}}{k}, \bar{\omega}=\frac{\omega v}{u_{0}^{2}} M=\frac{\sigma B_{0}^{2} \mathrm{v}}{\rho u_{0}^{2}}, \bar{t}=\frac{t u_{0}^{2}}{v}, \\
& G m=\frac{g \beta^{*} v\left(C_{w}-C_{\infty}\right)}{u_{0}{ }^{2}}, G r=\frac{g \beta v\left(T_{w}-T_{\infty}\right)}{u_{0}{ }^{3}}, \bar{C}=\frac{C-C_{\infty}}{C_{w}-C_{\infty}}, \theta=\frac{\left(T-T_{\infty}\right)}{\left(T_{w}-T_{\infty}\right)}, \mathrm{K}_{0}=\frac{v \mathrm{~K}_{\mathrm{c}}}{\mathrm{u}_{0}^{2}} .
\end{aligned}
$$

Here the dimensionless symbols used are as under

$\bar{u}$ - the dimensionless velocity, $\bar{w}$ - dimensionless velocity, $\theta$ - temperature, $\bar{C}$ - concentration, $G r$ - thermal Grashof number, $G m$ - mass Grashof number, $\mu$ - the coefficient of viscosity, $P r$ - the Prandtl number, $S c$ - the Schmidt number, $M$ - the magnetic parameter $K_{0}$ - the chemical reaction parameter.

The dimensionless forms of equations (1), (2), (3) and (4) are as follows

$$
\begin{aligned}
& \frac{\partial \bar{u}}{\partial \bar{t}}=\frac{\partial^{2} \bar{u}}{\partial \bar{y}^{2}}+G r \theta+G m \bar{C}-\frac{M(\bar{u}+m \bar{w})}{\left(1+m^{2}\right)}, \\
& \frac{\partial \bar{w}}{\partial \bar{t}}=\frac{\partial^{2} \bar{w}}{\partial \bar{y}^{2}}-\frac{M(\bar{w}-m \bar{u})}{\left(1+m^{2}\right)}, \\
& \frac{\partial \bar{C}}{\partial \bar{t}}=\frac{1}{S c} \frac{\partial^{2} \bar{C}}{\partial \bar{y}^{2}}-K_{0} \bar{C}, \\
& \frac{\partial \theta}{\partial \bar{t}}=\frac{1}{\operatorname{Pr}} \frac{\partial^{2} \theta}{\partial \bar{y}^{2}},
\end{aligned}
$$

The corresponding b. c. are

$\bar{t} \leq 0, \bar{u}=0, \bar{C}=0, \theta=0, \vec{w}=0$, for all values of $\overline{\mathrm{y}}$,

$\bar{t}>0, \bar{u}=\cos \bar{\omega} \bar{t}, \bar{w}=0, \theta=1, \bar{C}=\bar{t}$ at $\overline{\mathrm{y}}=0$,

$\bar{u} \rightarrow 0, \bar{C} \rightarrow 0, \theta \rightarrow 0, \bar{w} \rightarrow 0$ as $\bar{y} \rightarrow \infty$.

Dropping the bars and combining the equations (7) and (8), we get

$$
\begin{aligned}
& \frac{\partial q}{\partial t}=\frac{\partial^{2} q}{\partial y^{2}}+G r \theta+G m C-\left(\frac{M}{1+m^{2}}(1-m i)\right) q, \\
& \frac{\partial C}{\partial t}=\frac{1}{S c} \frac{\partial^{2} C}{\partial y^{2}}-K_{0} C \\
& \frac{\partial \theta}{\partial t}=\frac{1}{\operatorname{Pr}} \frac{\partial^{2} \theta}{\partial y^{2}}
\end{aligned}
$$

Here $q=u+i w$, and the corresponding boundary conditions are, 


$$
\begin{aligned}
& t \leq 0: q=0, \theta=0, C=0, \text { for all values of } \mathrm{y}, \\
& \mathrm{t}<0: q=\cos \omega t, \mathrm{w}=0, \theta=1, \mathrm{C}=\mathrm{t}, \text { at } \mathrm{y}=0, \\
& \mathrm{q} \rightarrow 0, \mathrm{C} \rightarrow 0, \theta \rightarrow 0, \text { asy } \rightarrow \infty .
\end{aligned}
$$

The solution of the above equations, obtained by the Laplace transformed method, is an under

$$
\begin{aligned}
& \theta=\operatorname{Erfc}\left[\frac{\sqrt{\operatorname{Pr}} y}{2 \sqrt{t}}\right], \\
& C=\frac{1}{4 \sqrt{S c K_{0}}} e^{-y \sqrt{S c K_{0}}}\left[B_{11}\right]\left(-S c y+2 t \sqrt{S c K_{0}}\right)+ \\
& e^{2 y \sqrt{S c K_{0}}}\left[B_{12}\right]\left(S c y+2 t \sqrt{S c K_{0}}\right) \text {, } \\
& q=\frac{1}{2} e^{-\sqrt{i \omega}}\left(P_{1}+P_{2}-P_{3}-P_{4}-P_{5}-P_{6}\right)+\frac{1}{2 a}\left(-e^{-\sqrt{a} y}\left(A_{0}\right)+e^{\frac{a t}{-1+\operatorname{Pr}}-y \sqrt{\frac{a t}{-1+\operatorname{Pr}}}}\left(A_{13}+e^{2 y \sqrt{\frac{a t}{-1+\operatorname{Pr}}}} A_{14}\right)\right) \\
& G r+\frac{1}{4\left(a-K_{0} S c\right)^{2}} y G m\left(\frac{2 e^{-\sqrt{a} y} A_{01}}{y}\left(1-a_{0}\right)+\sqrt{a} e^{-\sqrt{a} y}\left(A_{0}-e^{2 \sqrt{a} y} A_{12}\right)\right. \\
& +\frac{1}{y} 2 e^{\frac{a t}{-1+S c}-\frac{t K_{0} S c}{-1+S c}-y A_{17}}\left(-1-e^{2 y A_{17}}+A_{15}+e^{2 y A_{17}} A_{16}\right)[1-S c]+\frac{1}{y} 2 e^{-\sqrt{a y}} \\
& \left.\left(-1-e^{\sqrt{a y}}-A_{11}+e^{2 \sqrt{a y}} A_{12}\right) S c\left[1-t K_{0}\right]-\frac{1}{\sqrt{a}} e^{-\sqrt{a y}}\left(A_{0}-e^{2 \sqrt{a y}}\right) K_{0} S c\right) \\
& -\frac{1}{2 a}\left(e^{\frac{a t}{-1+\mathrm{Pr}}-y \sqrt{\frac{a t}{-1+\mathrm{Pr}}}}\left(1+A_{18}+e^{2 y \sqrt{\frac{a \mathrm{Pr}}{-1+\mathrm{Pr}}}} A_{19}\right)-2 \operatorname{Erfc}\left[\frac{y \sqrt{\mathrm{Pr}}}{2 \sqrt{t}}\right]\right) G r+ \\
& \frac{1}{4\left(a-K_{0} S c\right)^{2}} y G m \sqrt{S c}\left(-\frac{1}{\sqrt{K_{0}}} a e^{-y \sqrt{K_{0} S c}}\left(-1-e^{2 y \sqrt{K_{0} S c}}+A_{20}+e^{2 y \sqrt{K_{0} S c}} A_{21}\right)\right. \\
& +\frac{1}{y \sqrt{S c}} 2 e^{-y \sqrt{K_{0} S c}}\left(-1-e^{2 y \sqrt{K_{0} S c}}+A_{20}+e^{2 y \sqrt{K_{0} S c}} A_{21}\right)[1-t-S c]- \\
& \frac{1}{y \sqrt{S c}} 2 e^{\frac{a t}{-1+S c}-y A_{17} \frac{t K_{0} S c}{-1+S c}}\left(-1-e^{2 y A_{17}}-A_{22}+e^{2 y \sqrt{\frac{a-K_{0}}{-1+S c}} \sqrt{S c}} A_{23}\right)[1-S c]+e^{-y \sqrt{K_{0} S c}}
\end{aligned}
$$

More details of the notations are described in the appendix.

\section{Skin-friction}

The dimensionless skin-friction at the plate $y=0$ is computed by

$$
\left(\frac{d q}{d y}\right)_{y=0}=\tau_{x}+i \tau z .
$$

\section{Sherwood Number}

The dimensionless Sherwood number at the plate $y=0$ is computed by 


$$
\begin{aligned}
& S h=\left(\frac{\partial C}{\partial y}\right)_{y=0} \\
& S h=-\frac{1}{4} e^{-y \sqrt{S c K_{0}}}\left(B_{11}\left(-S c y+2 t \sqrt{S c K_{0}}\right)\right. \\
& +e^{2 y \sqrt{S c K_{0}}}\left(B_{12}\left(S c y+2 t \sqrt{S c K_{0}}\right)\right)+\frac{1}{4 \sqrt{S c K_{0}}} \\
& e^{-y \sqrt{S c K_{0}}}\left(-S c B_{11} e^{2 y \sqrt{S c K_{0}}} S c B_{12}\right)-\sqrt{\frac{S c}{\pi t}}\left(e^{B_{11}}(-S c+\right. \\
& \left.\left.2 t \sqrt{S c K_{0}}\right)+e^{-B_{12}{ }^{2}+2 y \sqrt{S c K_{0}}}\left(S c+2 t \sqrt{S c K_{0}}\right)\right)+ \\
& 2 e^{2 y \sqrt{S c K_{0}}} B_{12}\left(S c+2 t \sqrt{S c K_{0}}\right) .
\end{aligned}
$$

\section{Interpretation of results}

For computation, the values of parameters taken are:

$$
\begin{aligned}
& G r=10,20,30 ; M=1,3,5 ; m=1,1.5,2 ; \\
& \operatorname{Pr}=2,3,4 ; S c=2,3,5 ; \omega t=30^{\circ}, 45^{\circ}, 90^{\circ} \\
& K_{0}=1,10,20 ; G m=10,20,30 ; t=0.1,0.2,0.3 .
\end{aligned}
$$

Figures $2,3,5$, and 8 show that primary velocity $(u)$ increases when $G m, G r, m$, and $t$ are increased. In this case primary velocity increases with $m$, it means Hall current has increasing effect on the flow of the fluid along the plate. Figures 4, 6, 7, 9, and 10 show that primary velocity decreases when $M, P r, S c, \omega t$ and $K_{0}$ are increased. And figures 11, 12, 13, and 17 show that the secondary velocity increases when $G m, G r, M$, and $t$ are increased. Figures $14,15,16,18$ and 19 show that secondary velocity ( $w$ ) decreases when $m, P r, S c, \boldsymbol{\omega} t$ and $\mathrm{K}_{0}$ are increased. Here secondary velocity decreases when $m$ is increased; this implies that the Hall parameter slows down the transverse velocity. Figures 20 and 21 show that concentration decreases with increase in $K_{0}$ and $S c$, and figure 22 shows that it increases with increase in t. From table -1 it is observed that $\tau_{x}$ decreases with increase in $\operatorname{Pr}, M, S c, K_{0}$, and it increases with increase in $m, G m, G r, \omega t$ and $t . \tau_{z}$ increases with increase in $G r, t$, $G m$, and $M$, and it decreases when $S c, \operatorname{Pr}, K_{0}, \omega t$ and $m$ are increased. From table -2 it is observed that Sherwood number decreases with increase in $S c, K_{0}$, and $t$.

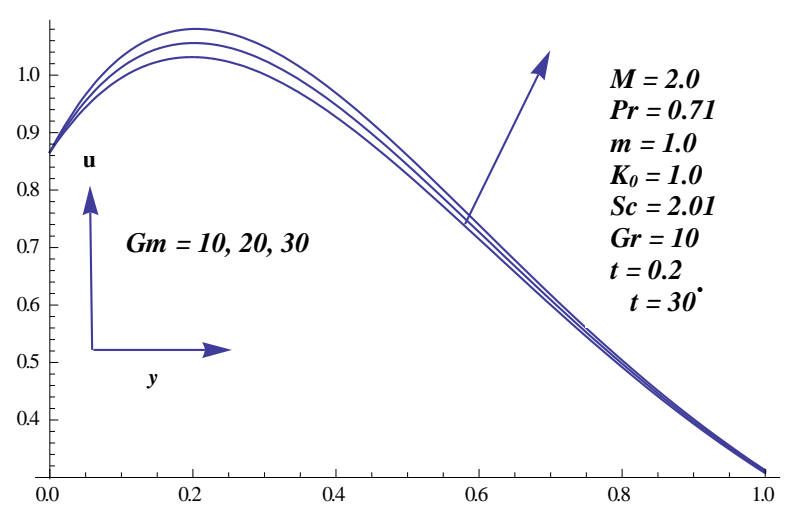

Figure 2: velocity u for different values of $G m$

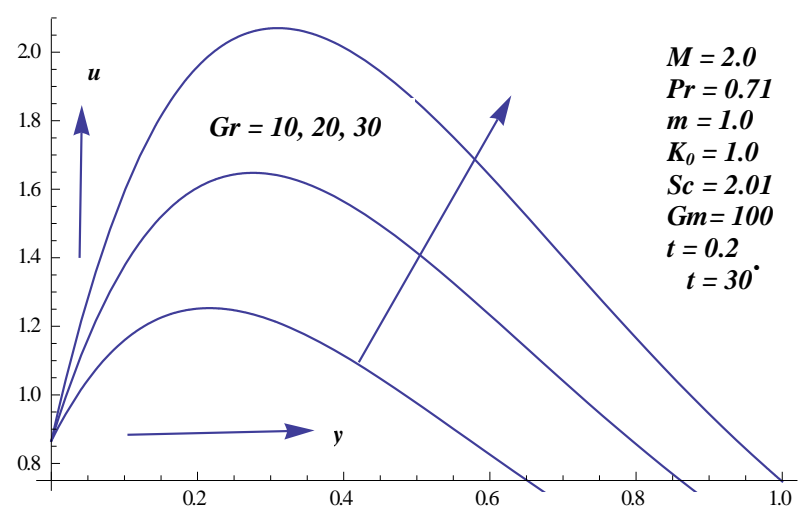

Figure 3: velocity u for different values of $G r$ 


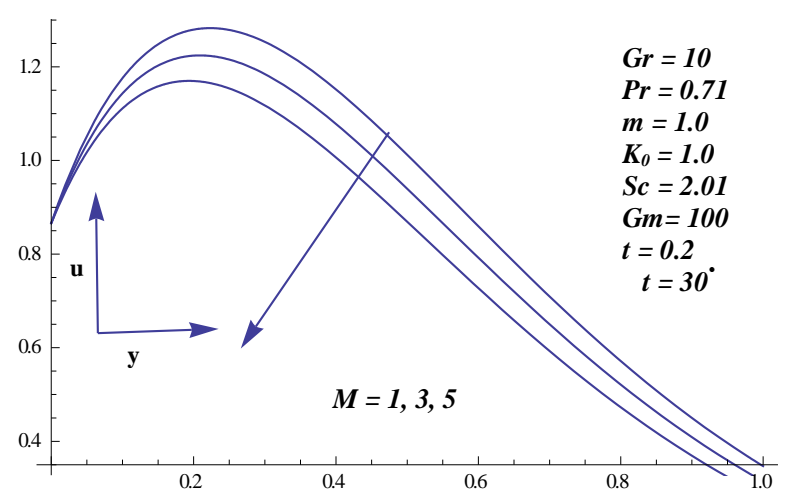

Figure 4: velocity u for different numeric values of $\mathrm{M}$

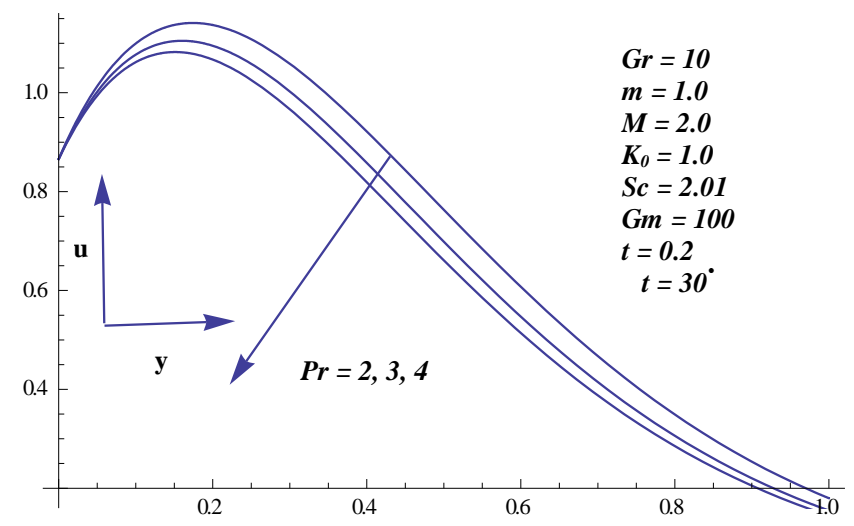

Figure 6: velocity u for different values of $\mathrm{Pr}$

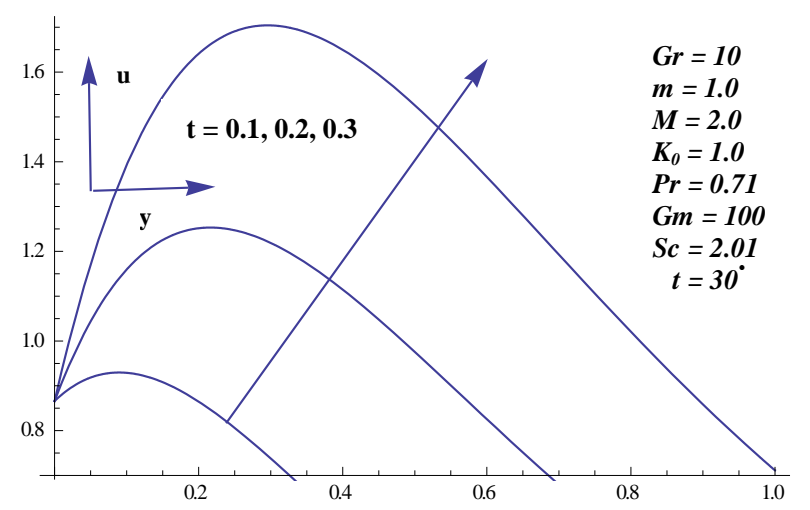

Figure 8: velocity u for different values of $t$

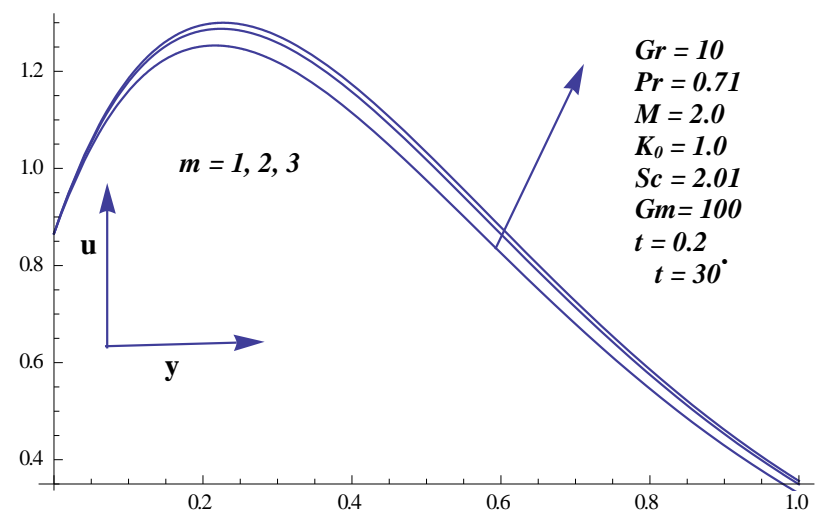

Figure 5: velocity u for different numeric values of $\mathrm{m}$

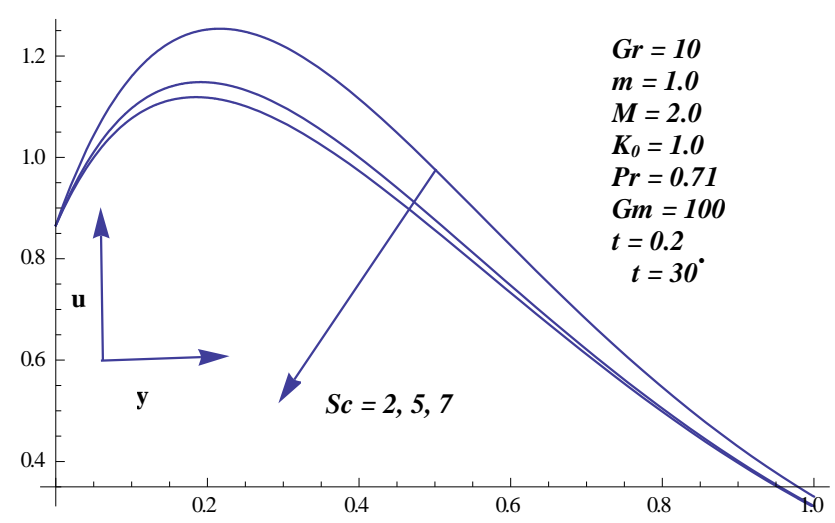

Figure 7: velocity u for different values of $S c$

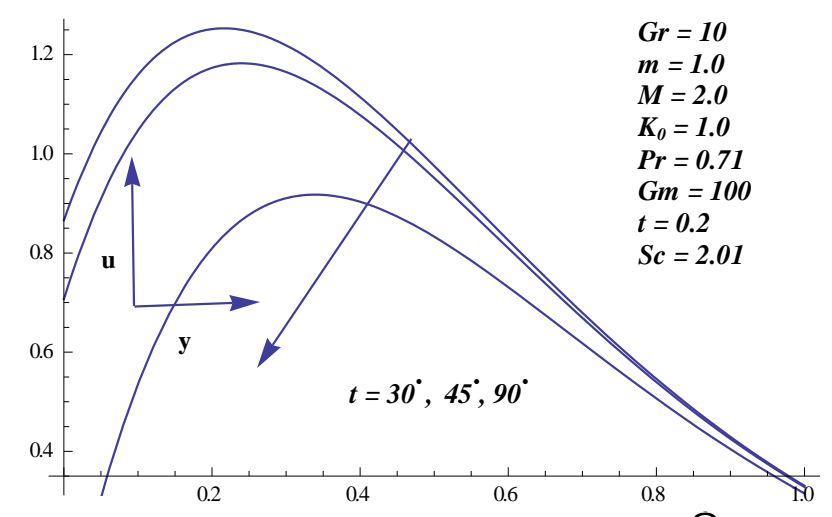

Figure 9: velocity u for different values of $\omega t$ 


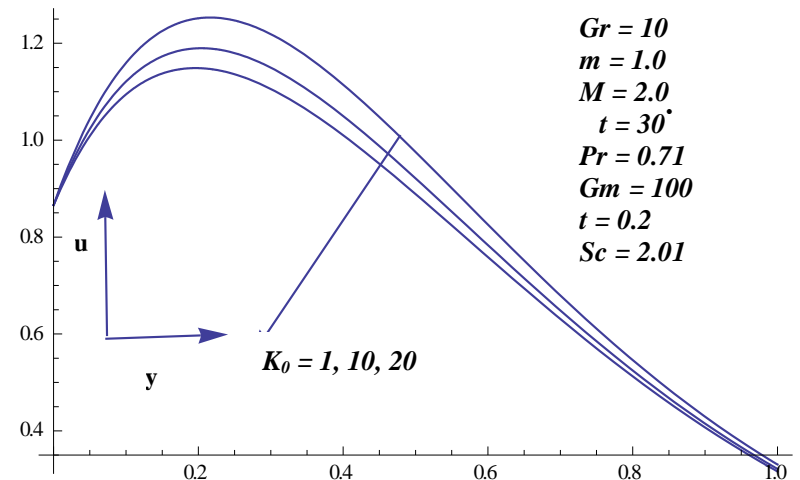

Figure 10: velocity $\mathrm{u}$ for different values of $\mathrm{K}_{0}$

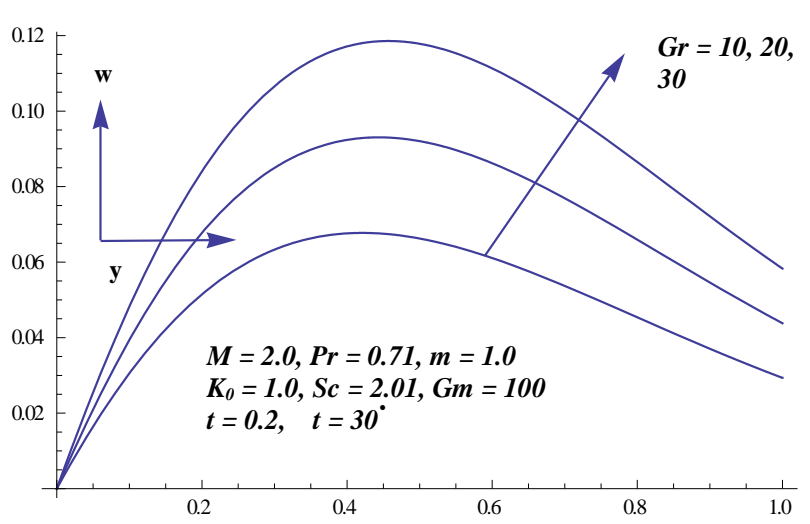

Figure 12: velocity w for different values of $G r$

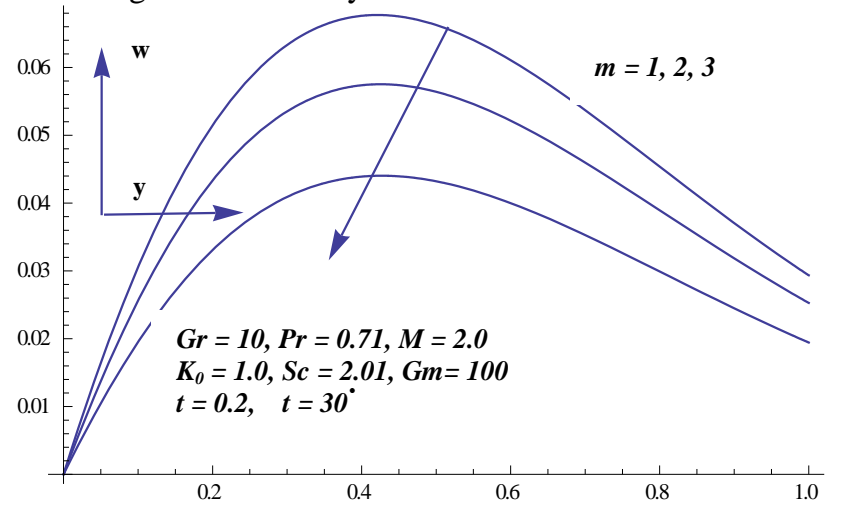

Figure 14: velocity w for different numeric values of $\mathrm{m}$

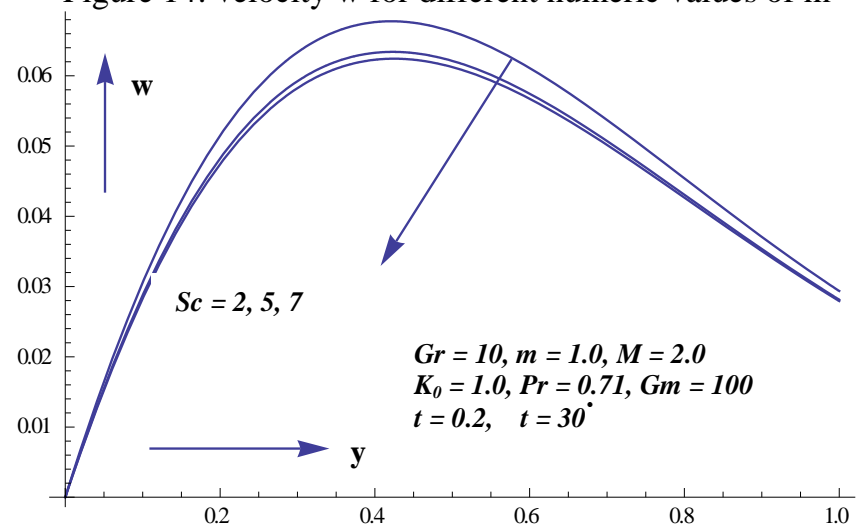

Figure 16: velocity w for different values of $S c$

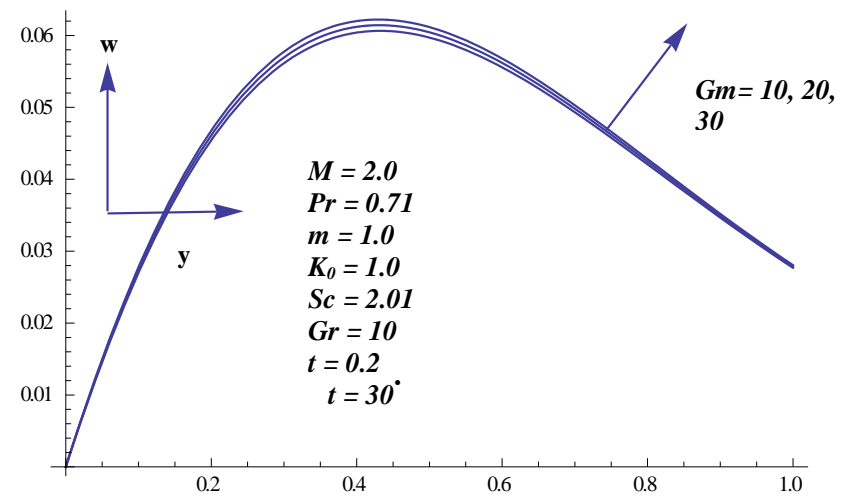

Figure 11: velocity w for different values of $\mathrm{Gm}$

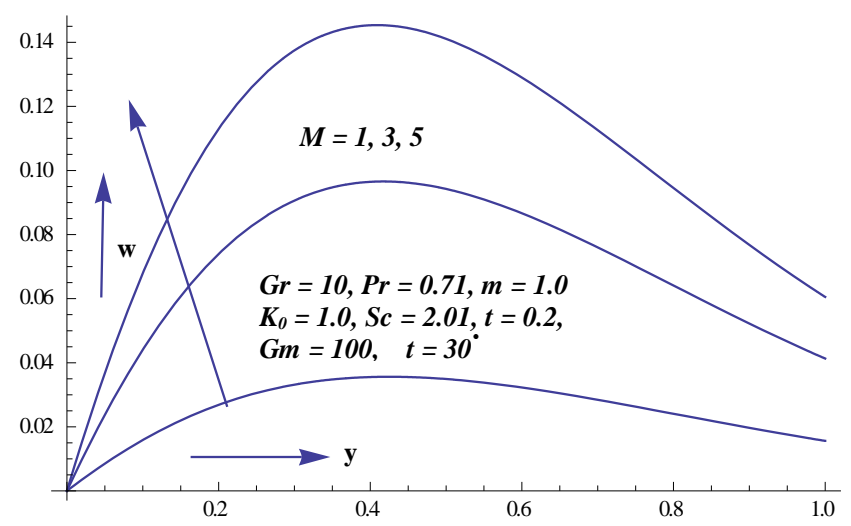

Figure 13: velocity w for different values of $M$

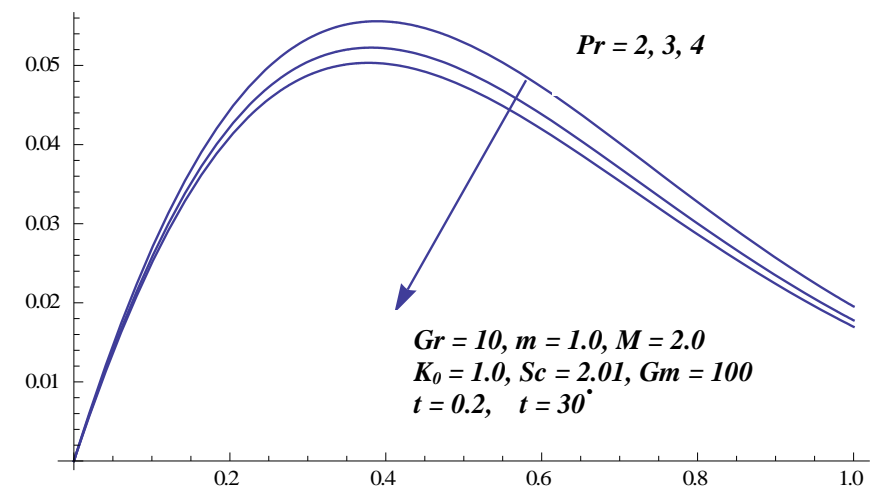

Figure 15: velocity w for different numeric values of $\mathrm{Pr}$

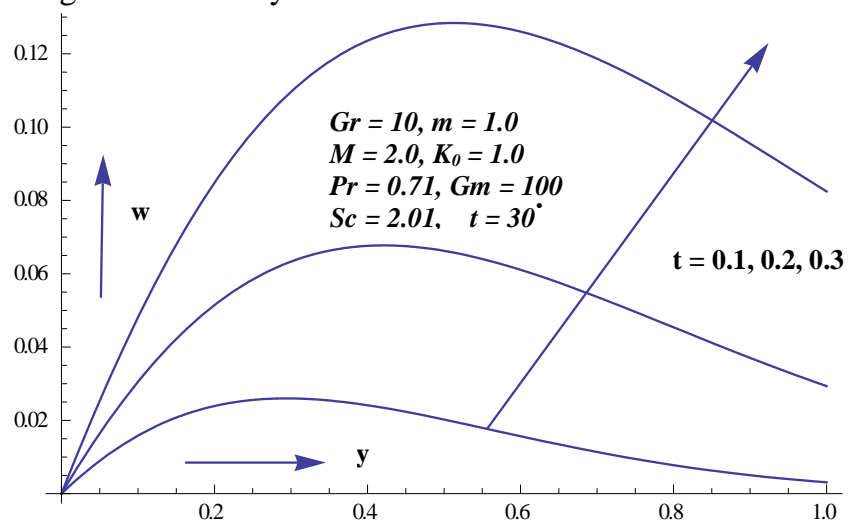

Figure 17: velocity w for different values of $t$ 


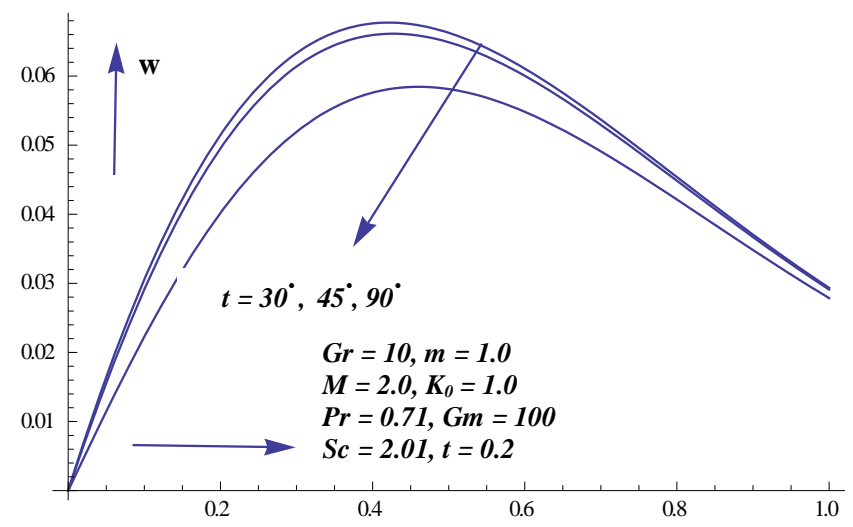

Figure 18: velocity w for different values of $\omega t$

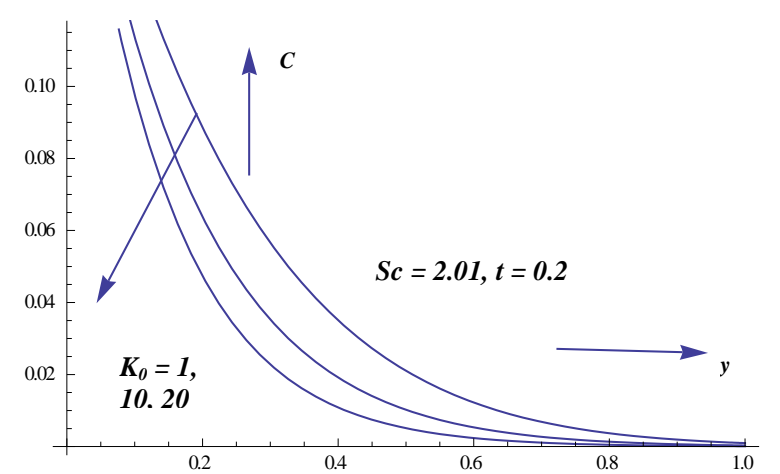

Figure 20: concentration profile for different values of $K_{0}$

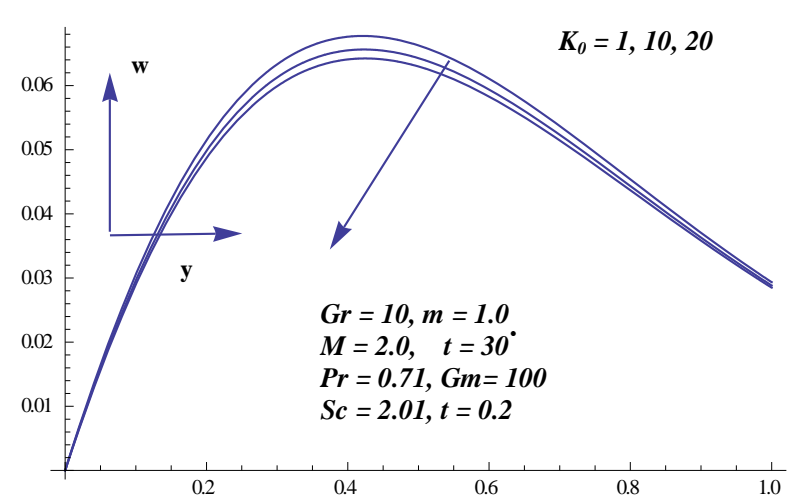

Figure 19: velocity w for different values of $\mathrm{K}_{0}$

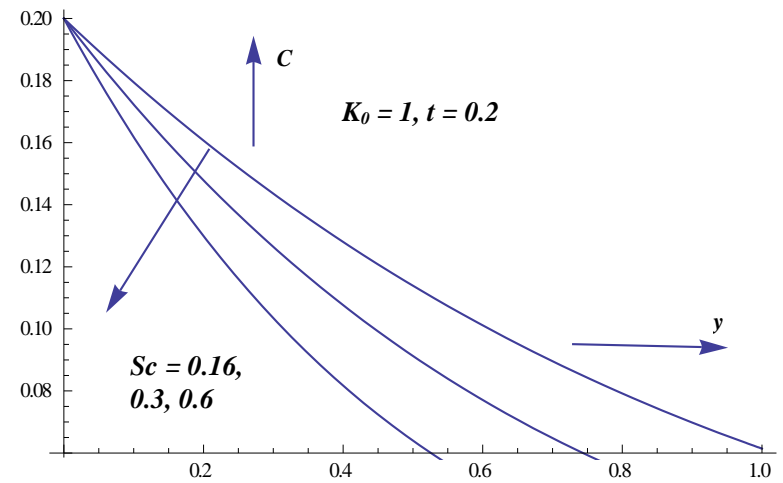

Figure 21: concentration profile for different values of $S c$

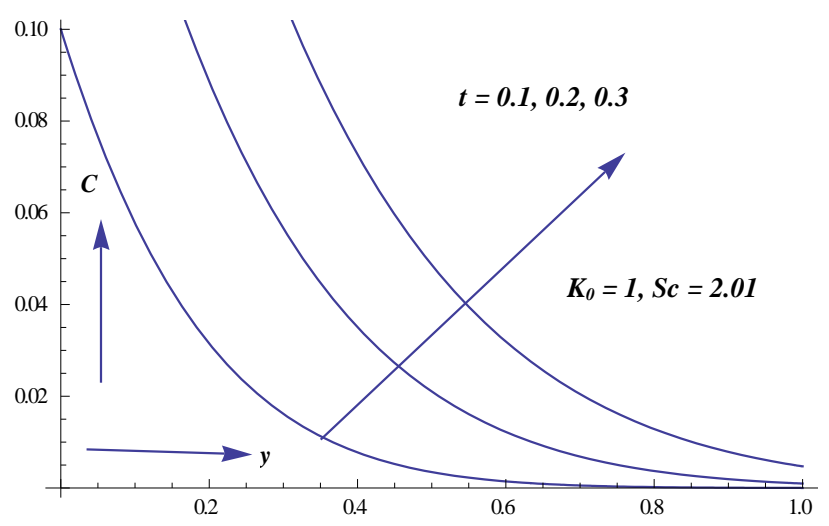

Figure 22: concentration profile for different values of $t$ 
Table 1. Skin friction

\begin{tabular}{|c|c|c|c|c|c|c|c|c|c|l|}
\hline $\boldsymbol{m}$ & $\boldsymbol{G r}$ & $\boldsymbol{G} \boldsymbol{M}$ & $\boldsymbol{M}$ & $\boldsymbol{S c}$ & $\boldsymbol{P r}$ & $\boldsymbol{\omega} \boldsymbol{t}$ & $\boldsymbol{t}$ & $\boldsymbol{K}_{\boldsymbol{o}}$ & $\tau_{x}$ & $\tau_{z}$ \\
\hline 1.0 & 10 & 100 & 2.0 & 2.01 & 2.00 & $30^{\circ}$ & 0.2 & 1.0 & 3.6594 & 0.3144 \\
\hline 1.0 & 10 & 100 & 2.0 & 2.01 & 3.00 & $30^{\circ}$ & 0.2 & 1.0 & 3.4289 & 0.3028 \\
\hline 1.0 & 10 & 100 & 2.0 & 2.01 & 4.00 & $30^{\circ}$ & 0.2 & 1.0 & 3.2716 & 0.2957 \\
\hline 1.0 & 10 & 100 & 2.0 & 2.01 & 0.71 & $30^{\circ}$ & 0.2 & 1.0 & 4.2666 & 0.3519 \\
\hline 1.0 & 20 & 100 & 2.0 & 2.01 & 0.71 & $30^{\circ}$ & 0.2 & 1.0 & 6.9065 & 0.4430 \\
\hline 1.0 & 30 & 100 & 2.0 & 2.01 & 0.71 & $30^{\circ}$ & 0.2 & 1.0 & 9.5463 & 0.5341 \\
\hline 2.0 & 10 & 100 & 2.0 & 2.01 & 0.71 & $30^{\circ}$ & 0.2 & 1.0 & 4.4861 & 0.2935 \\
\hline 3.0 & 10 & 100 & 2.0 & 2.01 & 0.71 & $30^{\circ}$ & 0.2 & 1.0 & 4.5437 & 0.2233 \\
\hline 1.0 & 10 & 10.0 & 2.0 & 2.01 & 0.71 & $30^{\circ}$ & 0.2 & 1.0 & 1.8590 & 0.3138 \\
\hline 1.0 & 10 & 20.0 & 2.0 & 2.01 & 0.71 & $30^{\circ}$ & 0.2 & 1.0 & 2.1265 & 0.3181 \\
\hline 1.0 & 10 & 30.0 & 2.0 & 2.01 & 0.71 & $30^{\circ}$ & 0.2 & 1.0 & 2.3941 & 0.3223 \\
\hline 1.0 & 10 & 100 & 1.0 & 2.01 & 0.71 & $30^{\circ}$ & 0.2 & 1.0 & 4.4547 & 0.1823 \\
\hline 1.0 & 10 & 100 & 3.0 & 2.01 & 0.71 & $30^{\circ}$ & 0.2 & 1.0 & 4.0799 & 0.5096 \\
\hline 1.0 & 10 & 100 & 5.0 & 2.01 & 0.71 & $30^{\circ}$ & 0.2 & 1.0 & 3.7125 & 0.7923 \\
\hline 1.0 & 10 & 100 & 2.0 & 2.00 & 0.71 & $30^{\circ}$ & 0.2 & 1.0 & 4.2705 & 0.3520 \\
\hline 1.0 & 10 & 100 & 2.0 & 5.00 & 0.71 & $30^{\circ}$ & 0.2 & 1.0 & 3.5904 & 0.3332 \\
\hline 1.0 & 10 & 100 & 2.0 & 7.00 & 0.71 & $30^{\circ}$ & 0.2 & 1.0 & 3.3659 & 0.3282 \\
\hline 1.0 & 10 & 100 & 2.0 & 2.01 & 0.71 & $30^{\circ}$ & 0.1 & 1.0 & 1.5484 & 0.2013 \\
\hline 1.0 & 10 & 100 & 2.0 & 2.01 & 0.71 & $30^{\circ}$ & 0.3 & 1.0 & 7.0431 & 0.5308 \\
\hline 1.0 & 10 & 100 & 2.0 & 2.01 & 0.71 & $30^{\circ}$ & 0.2 & 10 & 3.8433 & 0.3422 \\
\hline 1.0 & 10 & 100 & 2.0 & 2.01 & 0.71 & $30^{\circ}$ & 0.2 & 20 & 3.5459 & 0.3355 \\
\hline 1.0 & 10 & 100 & 2.0 & 2.01 & 0.71 & $45^{\circ}$ & 0.2 & 1.0 & 4.8078 & 0.3307 \\
\hline 1.0 & 10 & 100 & 2.0 & 2.01 & 0.71 & $90^{\circ}$ & 0.2 & 1.0 & 7.0341 & 0.2320 \\
\hline
\end{tabular}

Table - 2 Sherwood number

\begin{tabular}{|c|c|c|c|}
\hline $\boldsymbol{S c}$ & $\boldsymbol{K}_{\boldsymbol{0}}$ & $\boldsymbol{t}$ & $\boldsymbol{S h}$ \\
\hline 0.16 & 1.0 & 0.2 & -0.2150 \\
\hline 0.3 & 1.0 & 0.2 & -0.2944 \\
\hline 0.6 & 1.0 & 0.2 & -0.4164 \\
\hline 2.01 & 1.0 & 0.2 & -0.7621 \\
\hline 2.01 & 10 & 0.2 & -1.1182 \\
\hline 2.01 & 20 & 0.2 & -1.4264 \\
\hline 2.01 & 1.0 & 0.1 & -0.5225 \\
\hline 2.01 & 1.0 & 0.2 & -0.7621 \\
\hline 2.01 & 1.0 & 0.3 & -0.9613 \\
\hline
\end{tabular}

\section{Conclusion}

The conclusion is as under:

- $\mathrm{u}$ increases with the increase in thermal Grashof number, Hall parameter, mass Grashof number and time (Figures 3, 5, 2, and 8).

- $\quad \mathrm{u}$ decreases with the increase in Prandtl number, magnetic field, Schmidt number, chemical reaction and phase angle. (Figures 6, 4, 7, 10, and 9).

- w increases with increase in thermal Grashof number, mass Grashof number, time, and magnetic field parameter. (Figures $12,11,17$, and 13).

- w decreases with the increase in Hall parameter, Prandtl number, Schmidt number, chemical reaction and phase angle. $(14,15,16,19$, and 18).

- Skin-fraction (Table -1) $\tau_{x}$ decreases with increase in Schmidt number, Prandtl number, magnetic field parameter, chemical reaction. It increases with thermal Grashof number, mass Grashof number, Hall parameter, time, phase angle. 
$\tau_{z}$ increases with increase thermal Grashof number, mass Grashof number, time, and magnetic field. And it decreases with Prandtl number, Hall parameter, Schmidt number, chemical reaction, and phase angle.

- Concentration decreases with increase in $\mathrm{Sc}$ and $\mathrm{K}_{0}$, and it increases with increase in t. (Figures 21, 20, and 22).

- Sherwood number (Table - 2) decreases with increase in Sc, $\mathrm{K}_{0}$ and t.

\section{Appendix}

$$
\begin{aligned}
& P_{1}=e^{-y \sqrt{a-i \omega}}+e^{y \sqrt{a-i \omega}}, P_{2}=e^{-y \sqrt{a+i \omega}-2 i t \omega}+e^{y \sqrt{a+i \omega}+2 i t \omega}, P_{3}=e^{-y \sqrt{a-i \omega}} \operatorname{Erf}\left\{\frac{y-2 t \sqrt{a-i \omega}}{2 \sqrt{t}}\right\}, P_{4}=e^{-y \sqrt{a-i \omega}} \operatorname{Erf}\left\{\frac{y+2 t \sqrt{a-i \omega}}{2 \sqrt{t}}\right\}, \\
& P_{5}=e^{-y \sqrt{a-i \omega}+2 i t \omega} \operatorname{Erf}\left\{\frac{y-2 t \sqrt{a+i \omega}}{2 \sqrt{t}}\right\}, P_{6}=e^{y \sqrt{a-i \omega}+2 i t \omega} \operatorname{Erf}\left\{\frac{y+2 t \sqrt{a+i \omega}}{2 \sqrt{t}}\right\}, A_{0}=1+A_{11}+e^{2 \sqrt{a y}} A_{12}, A_{01}=1+e^{2 \sqrt{a} y}+A_{11}-e^{2 \sqrt{a y}} A_{12}, \\
& A_{11}=\operatorname{Erf}\left\{\frac{2 a \sqrt{t}-y}{2 \sqrt{t}}\right\}, A_{12}=\operatorname{Erf}\left\{\frac{2 a \sqrt{t}-y}{2 \sqrt{t}}\right\}, A_{13}=\operatorname{Erf}\left\{\frac{y-2 t \sqrt{a\left(1+\frac{1}{-1+\operatorname{Pr}}\right)}}{2 \sqrt{t}}\right\}, A_{14}=\operatorname{Erf}\left\{\frac{y+2 t \sqrt{a\left(1+\frac{1}{-1+\operatorname{Pr}}\right)}}{2 \sqrt{t}}\right\},
\end{aligned}
$$

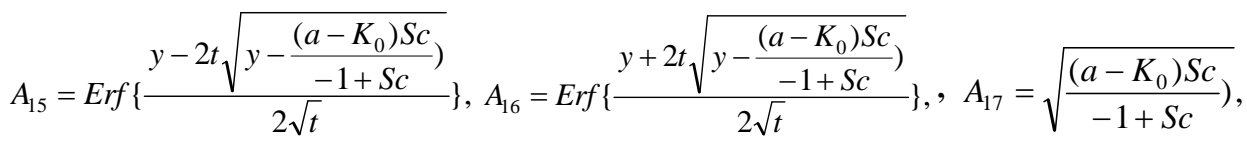

$$
\begin{aligned}
& A_{18}=\operatorname{Erf}\left\{\frac{2 t \sqrt{\frac{a}{-1+\operatorname{Pr}}}-y \sqrt{\mathrm{Pr}}}{2 \sqrt{t}}\right\}, A_{19}=\operatorname{Erf}\left\{\frac{2 t \sqrt{\frac{a}{-1+\operatorname{Pr}}}+y \sqrt{\operatorname{Pr}}}{2 \sqrt{t}}\right\}, A_{20}=\operatorname{Erf}\left\{\frac{2 t \sqrt{K_{0}}-y \sqrt{S c}}{2 \sqrt{t}}\right\}, A_{21}=\operatorname{Erf}\left\{\frac{2 t \sqrt{K_{0}}+y \sqrt{S c}}{2 \sqrt{t}}\right\}, \\
& A_{22}=\operatorname{Erf}\left\{\frac{2 t \sqrt{\frac{a-K_{0}}{-1+S c}}-y \sqrt{S c}}{2 \sqrt{t}}\right\}, A_{23}=\operatorname{Erf}\left\{\frac{2 t \sqrt{\frac{a-K_{0}}{-1+S c}}+y \sqrt{S c}}{2 \sqrt{t}}\right\}, \quad B_{11}=\operatorname{Erfc}\left\{\frac{\sqrt{S c} y-2 t \sqrt{K_{0}}}{2 \sqrt{t}}\right\}, \\
& B_{12}=\operatorname{Erfc}\left\{\frac{\sqrt{S c} y+2 t \sqrt{K_{0}}}{2 \sqrt{t}}\right\}, \quad a=\frac{M}{1+m^{2}}(1-i m) \text {. }
\end{aligned}
$$

\section{References}

Beg A. O. and Ghosh S. K., 2010, Analytical study of magneto hydro dynamic radiation-convection with surface temperature oscillation and secondary flow effects, International Journal of Applied Math \& Mechanics, Vol. 6, No. 6, pp. 1-22.

Chambre P. L. and Young J. D., 1958, On the diffusion of a chemically reactive species in a laminar boundary layer flow, Physics of Fluids, Vol. 1, pp. 48-54.

Datta N. and Jana R. N. 1976, Oscillatory magneto hydrodynamic flow past a flat plate will Hall effects, Journal of the Physical Society of Japan, Vol. 40, No. 5, pp. 1469-1474.

Ibrahim S. Y., and Makinde O. D., 2010, Chemically reacting MHD boundary layer flow of heat and mass transfer over a moving vertical plate with suction, Scientific Research and Essays, Vol. 5, No. 19, pp. 2875-2882.

Ibrahim S.M., 2014, Effects of chemical reaction on dissipative radiative MHD flow through a porous medium over a nonisothermal stretching sheet, Hindawi Publishing Corporation Journal of Industrial Mathematics, Vol. 2014,10 p., http://dx.doi.org/10.1155/2014/243148

Kumar J. Prathap, Umavathi J.C. and Karuna Prasad M., 2016, Effect of viscous dissipation on mixed convection flow in a vertical double passage channel using Robin boundary conditions, International Journal of Engineering, Science and Technology Vol. 8, No. 3, pp. 27-47.

Mishra S.R., Dash G.C., M. Acharya, 2012, Heat transfer in viscous free convective fluctuating MHD flow through porous media past a vertical porous plate with variable temperature, Mathematical Theory and Modeling, Vol. 2, No. 6, pp. 1-14.

Nguyen M. T., Aly A. M, Lee S-W, 2016 "Unsteady natural convection heat transfer in a nanofluid-filled square cavity with various heat source conditions", Advances in Mechanical Engineering, Vol. 8, No. 5, pp. 1-18.

Rajput U.S. and Kanaujia N., 2016, MHD flow past a vertical plate with variable temperature and mass diffusion in the presence of Hall current, International Journal of Applied Science and Engineering, Vol. 14, No. 2, pp. 115-123.

Rajput U.S. and Kumar G., 2016, Unsteady MHD flow in porous media past over exponentially accelerated inclined plate with variable wall temperature and mass transfer along with Hall current, International Journal of Engineering, Science and Technology vol. 8, No. 2, pp. 1-10. 
Sato H., 1961, The Hall effect in the viscous flow of ionized gas between parallel plates under transverse magnetic field, Journal of the Physical Society of Japan, Vol. 16, No. 7, 1427-1433.

Satya Narayana P.V., Venkateswarlu B., Devika B., 2016 Chemical reaction and heat source effects on MHD oscillatory flow in an irregular channel, Ain Shams Engineering Journal, Vol. 7, pp. 1079-1088.

Takabi B., Salehi S., 2014, Augmentation of the heat transfer performance of a sinusoidal corrugated enclosure by employing hybrid nanofluid, Advances in Mechanical Engineering, Vol. 6, 147059

Yamanishi T., 1962, Effect of Hall current on the steady hydromagnetic flow between two parallel a plates, in: Proc. 17th Annual Meeting, Phys. Soc. Japan, Vol. 5, Osaka, Japan, p. 29.

Biographical notes

Dr U. S. Rajput is a permanent faculty member in the Department of Mathematics and Astronomy, Lucknow University, India.

Neetu Kanaujia is research scholar in the Department of Mathematics and Astronomy, Lucknow University, India.

Received October 2016

Accepted December 2016

Final acceptance in revised form December 2016 